\title{
A result on the existence of common quadratic Lyapunov functions for pairs of stable discrete-time LTI systems
}

\author{
Oliver Mason ${ }^{1}$ \\ Hamilton Institute \\ NUI Maynooth \\ Ireland
}

\begin{abstract}
This paper deals with the existence of weak and strong common quadratic Lyapunov functions (CQLFs) for pairs of stable discrete-time linear time-invariant (LTI) systems. The main result of the paper provides a simple characterisation of pairs of such systems for which a weak CQLF of a given form exists but for which no strong CQLF exists. An application of this result to second order discrete-time LTI systems is presented.
\end{abstract}

\section{Introduction}

In recent years, the area of systems theory and control has witnessed a considerable growth of interest in systems characterised by a combination of continuous dynamics and logic-based switching. A major issue for such systems has been the determination of easily verifiable and interpretable conditions that guarantee stability. For an overview of some recent approaches to this issue see [1], [2], [3], [4], [5], [6], [7]. In this context the problem of the existence or non-existence of a common quadratic Lyapunov function (CQLF) for a family of linear time-invariant (LTI) systems is of considerable interest. There is already a substantial body of literature dedicated to this question for both discrete-time and continuous-time systems $[8],[9],[10],[11],[12]$, [13], [7]. The main result presented here is concerned with the CQLF existence problem for a family of two discrete-time LTI systems.

\section{Notation and Preliminaries}

Throughout $\mathbb{R}$ and $\mathbb{C}$ will denote the fields of real and complex numbers respectively and $\mathbb{R}^{n \times n}\left(\mathbb{C}^{n \times n}\right)$ denotes the space of $n \times n$ matrices with real (complex) entries. For a matrix $A$ in $\mathbb{R}^{n \times n}, A^{T}$ denotes its transpose, $\operatorname{det}(A)$ its determinant and $a_{i j}$ the entry in the $(i, j)$ position of $A$. Similarly for a vector $x$ in $\mathbb{R}^{n}, x_{i}$

\footnotetext{
1 oliver.mason@may.ie

${ }^{2}$ robert.shorten@may.ie
}

\author{
Robert Shorten ${ }^{2}$ \\ Hamilton Institute \\ NUI Maynooth \\ Ireland
}

denotes the $i^{\text {th }}$ component of $x$. A matrix $A \in \mathbb{R}^{n \times n}$ is said to be symmetric if $A=A^{T}$. The notation $P>0$ $(P \geq 0)$ is used to denote that the matrix $P$ is positive (semi-)definite with $P<0,(P \leq 0)$ meaning that $-P>0(-P \geq 0)$. Recall that $A \in \mathbb{R}^{n \times n}$ is said to be Schur-stable (Hurwitz) if the eigenvalues of $A$ all lie in the open unit disk (open left half plane) in $\mathbb{C}$.

We note that a matrix $A \in \mathbb{R}^{n \times n}$ is Schur-stable if and only if the associated discrete-time LTI system

$$
\Sigma_{A}: x(k+1)=A x(k)
$$

is asymptotically stable [14].

In the spirit of [15], we now define strong and weak CQLFs for a set of stable discrete-time LTI systems.

\section{Strong and weak CQLFs:}

Consider the set of stable discrete-time LTI systems

$$
\Sigma_{A_{i}}: x(k+1)=A_{i} x(k), 1 \leq i \leq M .
$$

where the $A_{i}$ are Schur-stable matrices in $\mathbb{R}^{n \times n}$. If there is a simultaneous solution $P=P^{T}>0$ to the discrete-time Lyapunov inequalities ${ }^{1}$

$$
A_{i}^{T} P A_{i}-P=-Q_{i}<0 \quad 1 \leq i \leq M
$$

then the scalar function $V(x)=x^{T} P x$ is said to be a strong $C Q L F$ for the systems $\Sigma_{A_{i}}$. If $M=1$, then $V(x)$ is said to be a strong quadratic Lyapunov function for the system $\Sigma_{A_{1}}$.

- Similarly, if $P=P^{T}>0$ simultaneously satisfies the non-strict inequalities

$$
A_{i}^{T} P A_{i}-P=-Q_{i} \leq 0 \quad 1 \leq i \leq M
$$

we say that $V(x)=x^{T} P x$ is a weak $C Q L F$ for the systems $\Sigma_{A_{i}}$. A weak quadratic Lyapunov function for a single system is defined in the obvious manner.

The notion of a matrix pencil, defined below, will be convenient for expressing our later results.

${ }^{1}$ referred to as the Stein inequalities by some authors 
The matrix pencil $\sigma_{\gamma[0, \infty)}\left[A_{1}, A_{2}\right]$ :

Given $A_{1}, A_{2} \in \mathbb{R}^{n \times n}$, the matrix pencil $\sigma_{\gamma[0, \infty)}\left[A_{1}, A_{2}\right]$ is the parameterised family of matrices

$$
\sigma_{\gamma[0, \infty)}\left[A_{1}, A_{2}\right]=\left\{A_{1}+\gamma A_{2}: \gamma \in[0, \infty)\right\} .
$$

We say that the pencil is non-singular if $A_{1}+\gamma A_{2}$ is non-singular for all $\gamma \geq 0$. Otherwise the pencil is said to be singular.

\section{Some Preliminary Lemmas}

In this section, we state without proof a number of lemmas that are needed to establish the results of the following sections. For details of the proofs of these lemmas, consult the report [16]. The following wellknown lemma provides a convenient test for singularity of a matrix pencil.

Lemma 2.1 : Let $A_{1}, A_{2}$ be non-singular matrices in $\mathbb{R}^{n \times n}$. The pencil $\sigma_{\gamma(0, \infty)}\left[A_{1}, A_{2}\right]$ is singular if and only if the matrix product $A_{1} A_{2}^{-1}$ has a negative (real) eigenvalue.

We next record the simple observation that the quadratic Lyapunov functions for the stable discretetime LTI systems $\Sigma_{A}$ and $\Sigma_{-A}$ coincide. This relates to the result in [17] identifying the quadratic Lyapunov functions for $\Sigma_{A}$ and $\Sigma_{A-1}$ for continuous-time systems. A related observation was also made in [18].

Lemma 2.2 : Consider the stable discrete-time LTI systems

$$
\begin{aligned}
\Sigma_{A}: x(k+1) & =A x(k) \\
\Sigma_{-A}: x(k+1) & =(-A) x(k) .
\end{aligned}
$$

Then, any quadratic Lyapunov function for $\Sigma_{A}$ is also a quadratic Lyapunov function for $\Sigma_{-A}$.

Lemma 2.3: Let $u, v, x, y \in \mathbb{R}^{\mathrm{n}}$ be any four non-zero vectors. There exists a non-singular $T \in \mathbb{R}^{n \times n}$ such that each component of the vectors $T u, T v, T x, T y$ is non-zero.

The next result (Lemma 2.4) establishes a convenient relationship between two parameterizations of the same hyperplane in the space of symmetric matrices in $\mathbb{R}^{n \times n}$.

Lemma 2.4 : Let $x, y, u, v$ be 4 non-zero vectors in $\mathrm{IR}^{n}$. Suppose that there is some $k>0$ such that for all $n \times n$ real symmetric matrices $H$

$$
x^{T} H y=-k u^{T} H v .
$$

Then either

$$
x=\alpha u \text { for some real scalar } \alpha, \text { and } y=-\left(\frac{k}{\alpha}\right) v
$$

or

$$
x=\beta v \text { for some real scalar } \beta \text { and } y=-\left(\frac{k}{\beta}\right) u .
$$

\section{Main results}

The principal result of this paper concerns two stable discrete-time LTI systems for which no strong CQLF exists but for which a weak CQLF exists with each of the $Q_{i}, i \in\{1,2\}$ in (3) of rank $n-1$. In Theorem 3.1 we provide a simple algebraic characterisation of this situation. The result is of interest for any class of systems where the transition from the existence of a CQLF to the non-existence of a CQLF passes through the situation described in the theorem.

Remark: It is possible to show that for any Schurstable matrix $A \in \mathbb{R}^{n \times n}$, the set of matrices $P=P^{T}$ satisfying

$$
A^{T} P A-P=-Q \quad Q \geq 0, \operatorname{rank}(Q)=n-1
$$

is dense in the set of matrices satisfying

$$
A^{T} P A-A=-Q \quad Q \geq 0, \operatorname{det}(Q)=0 .
$$

This indicates that the situation described in the theorem is potentially of great importance in providing insight into the existence question for strong and weak CQLFs.

Before stating Theorem 3.1, we introduce the notation $C(A)=(A-I)(A+I)^{-1}$ for $A$ in $\mathbb{R}^{n \times n}$. Note that $C(A)$ is well-defined for any Schur-stable $A$.

Theorem 3.1 : Let $\Sigma_{A_{1}}, \Sigma_{A_{2}}$ be two stable discretetime LTI systems such that a solution $P=P^{T}>0$ exists to the non-strict Lyapunov equations

$$
\begin{aligned}
& A_{1}^{T} P A_{1}-P=-Q_{1} \leq 0, \\
& A_{2}^{T} P A_{2}-P=-Q_{2} \leq 0,
\end{aligned}
$$

for some positive semi-definite matrices $Q_{1}, Q_{2}$ both of rank $n-1(n \geq 2)$. Furthermore suppose that the systems $\Sigma_{A_{1}}, \Sigma_{A_{2}}$ do not have a strong CQLF. Under these conditions, at least one of the pencils $\sigma_{\gamma[0, \infty)}\left[C\left(A_{1}\right), C\left(A_{2}\right)\right], \sigma_{\gamma[0, \infty)}\left[C\left(A_{1}\right), C\left(A_{2}\right)^{-1}\right]$ is singular, and equivalently, at least one of the matrix products $C\left(A_{1}\right) C\left(A_{2}\right)$ and $C\left(A_{1}\right) C\left(A_{2}\right)^{-1}$ has a real negative eigenvalue.

Proof: As $Q_{1}$ and $Q_{2}$ are of rank $n-1$, there are non-zero vectors $x_{1}, x_{2}$ such that

$$
x_{i}^{T} Q_{i} x_{i}=0, i=1,2
$$

The proof of Theorem 3.1 is split into two main stages. 
Stage 1: The first stage in the proof is to show that if there exists a real symmetric matrix $H$ satisfying

$$
\begin{aligned}
& x_{1}^{T}\left(A_{1}^{T} H A_{1}-H\right) x_{1}<0 \\
& x_{2}^{T}\left(A_{2}^{T} H A_{2}-H\right) x_{2}<0
\end{aligned}
$$

then $\Sigma_{A_{1}}, \Sigma_{A_{2}}$ would have a strong CQLF.

So assume that there is some $H$ satisfying (8), (9), and consider the set

$$
\Omega_{1}=\left\{x \in \mathbb{R}^{n}:\|x\|=1, x^{T}\left(A_{1}^{T} H A_{1}-H\right) x \geq 0\right\} .
$$

(Here $\|x\|$ is the usual Euclidean norm on $\mathbb{R}^{n}$.)

We shall show that there is a positive constant $C_{1}>$ 0 such that $A_{1}^{T}\left(P+\delta_{1} H\right) A_{1}-\left(P+\delta_{1} H\right)$ is negative definite provided that $0<\delta_{1}<C_{1}$.

Firstly suppose that $\Omega_{1}$ was empty. Then $A_{1}^{T}(P+$ $\left.\delta_{1} H\right) A_{1}-\left(P+\delta_{1} H\right)$ is negative definite for any $\delta_{1}>0$. So any positive constant $C_{1}$ will work in this case.

Now, assume that the set $\Omega_{1}$ is non-empty. The function that takes $x$ to $x^{T}\left(A_{1}^{T} H A_{1}-H\right) x$ is continuous. Thus $\Omega_{1}$ is closed and bounded, hence compact. Furthermore $x_{1}$ (or any non-zero multiple of $x_{1}$ ) is not in $\Omega_{1}$ and thus $x^{T}\left(A_{1}^{T} P A_{1}-P\right) x$ is strictly negative on $\Omega_{1}$.

Let $M_{1}$ be the maximum value of $x^{T}\left(A_{1}^{T} H A_{1}-H\right) x$ on $\Omega_{1}$, and let $M_{2}$ be the maximum value of $x^{T}\left(A_{1}^{T} P A_{1}-\right.$ $P) x$ on $\Omega_{1}$. Then by the final remark in the previous paragraph, $M_{2}<0$. Choose any constant $\delta_{1}>0$ such that

$$
\delta_{1}<\frac{\left|M_{2}\right|}{M_{1}+1}
$$

and consider the matrix

$$
P+\delta_{1} H \text {. }
$$

By separately considering the cases $x \in \Omega_{1}$ and $x \notin$ $\Omega_{1},\|x\|=1$, it is easy to see that for all non-zero vec- . tors $x$ of norm 1

$$
x^{T}\left(A_{1}^{T}\left(P+\delta_{1} H\right) A_{1}-\left(P+\delta_{1} H\right)\right) x<0
$$

provided $0<\delta_{1}<\frac{\left|M_{2}\right|}{M_{1}+1}$. Let $C_{1}$ denote the value $\frac{\left|M_{2}\right|}{M_{1}+1}$. Thus we have shown that there is some positive constant $C_{1}$ such that $A_{1}^{T}\left(P+\delta_{1} H\right) A_{1}-\left(P+\delta_{1} H\right)$ is negative definite provided that $0<\delta_{1}<C_{1}$.

Now the same argument can be used to guarantee the existence of a positive constant $C_{2}$ such that

$$
x^{T}\left(A_{2}^{T}\left(P+\delta_{1} H\right) A_{2}-\left(P+\delta_{1} H\right)\right) x<0 .
$$

for all non-zero $x$ provided we choose $0<\delta_{1}<C_{2}$. So, if we choose $\delta>0$ less than the minimum of $C_{1}, C_{2}$, we would have a real symmetric matrix

$$
P_{1}=P+\delta H
$$

satisfying (2) with $Q_{1}, Q_{2}>0$. This implies that $P_{1}>$ 0 [14] and thus $V(x)=x^{T} P_{1} x$ would be a strong CQLF for $\Sigma_{A_{1}}, \Sigma_{A_{2}}$.

Stage 2: So, under our assumptions there is no real symmetric matrix $H$ such that

$$
\begin{aligned}
& x_{1}^{T}\left(A_{1}^{T} H A_{1}-H\right) x_{1}<0 \\
& x_{2}^{T}\left(A_{2}^{T} H A_{2}-H\right) x_{2}<0 .
\end{aligned}
$$

Thus, the two linear functionals defined on the space of real symmetric matrices in $\mathbb{R}^{n \times \pi}$ by

$$
H \rightarrow x_{i}^{T}\left(A_{i}^{T} H A_{i}-H\right) x_{i} \quad i \in\{1,2\}
$$

must have the same kernel. This together with the fact that there is no $H$ satisfying (10), (11) implies that there is some positive constant $k$ such that

$$
x_{1}^{T}\left(A_{1}^{T} H A_{1}-H\right) x_{1}=-k x_{2}^{T}\left(A_{2}^{T} H A_{2}-H\right) x_{2}
$$

for all real symmetric matrices $H$.

Expanding the expression

$$
\left(A_{i} x_{i}-x_{i}\right)^{T} H\left(A_{i} x_{i}+x_{i}\right)
$$

and noting that, for symmetric $H$,

$$
x_{i}^{T} A_{i}^{T} H x_{i}-x_{i}^{T} H A_{i} x_{i}=0,
$$

we see that the two expressions

$$
\begin{aligned}
& x_{i}^{T}\left(A_{i}^{T} H A_{i}-H\right) x_{i} \\
& \left(A_{i} x_{i}-x_{i}\right)^{T} H\left(A_{i} x_{i}+x_{i}\right)
\end{aligned}
$$

are identical for all symmetric $H \in \mathbb{R}^{n \times n}$ for $i=1,2$.

Combining this fact with (12) and applying Lemma 2.4 now shows that either

$$
\begin{aligned}
& \left(A_{1} x_{1}+x_{1}\right)=\alpha\left(A_{2} x_{2}+x_{2}\right) \\
& \left(A_{1} x_{1}-x_{1}\right)=-\frac{k}{\alpha}\left(A_{2} x_{2}-x_{2}\right)
\end{aligned}
$$

or

$$
\begin{aligned}
& \left(A_{1} x_{1}+x_{1}\right)=\alpha\left(A_{2} x_{2}-x_{2}\right) \\
& \left(A_{1} x_{1}-x_{1}\right)=-\frac{k}{\alpha}\left(A_{2} \dot{x}_{2}+x_{2}\right)
\end{aligned}
$$

In the first case (15), we have

$$
x_{1}=\alpha\left(A_{1}+I\right)^{-1}\left(A_{2}+I\right)^{t} x_{2} .
$$

and substituting this into the second identity in (15) yields

$$
\left(A_{1}-I\right)\left(A_{1}+I\right)^{-1}\left(A_{2}+I\right) x_{2}=-\frac{k}{\alpha^{2}}\left(A_{2}-I\right) x_{2}
$$


Letting $y=\left(A_{2}+I\right) x_{2}$ we see that

$$
\left(C\left(A_{1}\right)+\frac{k}{\alpha^{2}} C\left(A_{2}\right)\right) y=0
$$

and hence the pencil $\sigma_{\gamma[0, \infty)}\left[C\left(A_{1}\right), C\left(A_{2}\right)\right]$ is singular and the product $C\left(A_{1}\right) C\left(A_{2}\right)^{-1}$ has a negative eigenvalue. A similar argument shows that in the case (16), the pencil $\sigma_{\gamma[0, \infty)}\left\{C\left(A_{1}\right), C\left(A_{2}\right)^{-1}\right\}$ is singular and the product $C\left(A_{1}\right) C\left(A_{2}\right)$ has a negative eigenvalue. This completes the proof of Theorem 3.1 .

Remarks:

(i) It is worth noting that the so-called bilinear transform, $C(A)=(A-I)(A+I)^{-1}[19,20]$, appears naturally in the course of the proof of Theorem 3.1 .

(ii) The positive definite $P$ assumed in the statement of Theorem 3.1 need only be semi-definite. The conclusions of the theorem are still valid in this case.

(iii) A crucial point in relation to Theorem 3.1 is that there is a hyperplane separating the two convex sets $\left\{P: A_{i}^{T} P A_{i}-P<0\right\}, i=1,2$. Essentially, the effect of the rank $n-1$ assumption is that this hyperplane is unique.

\section{Second order systems}

In this section we present an example to illustrate the use of Theorem 3.1 .

\section{Example: Second order systems}

Let $\Sigma_{A_{1}}$ and $\Sigma_{A_{2}}$ be stable discrete-time LTI systems with $A_{1}, A_{2} \in \mathbb{R}^{2 \times 2}$. We note the following readily verifiable facts.

(a) If $P=P^{T}$ satisfies $A^{T} P A-P=-Q \leq 0$, then $C(A)^{T} P+P C(A)=-Q^{\prime} \leq 0$ with $Q^{\prime}=2(A+$ $I)^{-T} Q(A+I)^{-1}$ having the same rank as $Q$.

(b) If a CQLF exists for $\Sigma_{A_{1}}$ and $\Sigma_{A_{2}}$, then a CQLF (in the continuous-time sense) exists for the systems $\Sigma_{C\left(A_{1}\right)}, \Sigma_{C\left(A_{2}\right)}$, and the pencils $\sigma_{\gamma[0, \infty)}\left[C\left(A_{1}\right), C\left(A_{2}\right)\right]$ and

$\sigma_{\gamma[0, \infty)}\left[C\left(A_{1}\right), C\left(A_{2}^{-1}\right)\right]$ must consist entirely of Hurwitz matrices.

(c) If a CQLF does not exist for $\Sigma_{A_{1}}$ and $\Sigma_{A_{2}}$ then a CQLF (in the continuous-time sense) does not exist for the continuous-time systems $\Sigma_{C\left(A_{1}\right)}$, $\Sigma_{C\left(A_{2}\right)}$. However by choosing $d>0$ sufficiently large, we can ensure that a CQLF (in the continuous-time sense) exists for $\Sigma_{C\left(A_{1}\right)-d I}$ and $\Sigma_{C\left(A_{2}\right)}$. A continuity argument can be employed to show that there is some $d_{1}$ with $0<d_{1}<d$ such that $C^{-1}\left(C\left(A_{1}\right)-d_{i} I\right)$ and $A_{2}$ satisfy Theorem 3.1, and thus one of the pencils $\sigma_{\gamma[0, \infty)}\left[C\left(A_{1}\right)-d_{1} I, C\left(A_{2}\right)\right]$ and $\sigma_{\gamma[0, \infty)}\left[C\left(A_{1}\right)-d_{1} I, C\left(A_{2}\right)^{-1}\right]$ is necessarily singular. Hence, it follows that one of the pencils $\sigma_{\gamma \mid 0, \infty)}\left[C\left(A_{1}\right), C\left(A_{2}\right)\right], \sigma_{\gamma \mid 0, \infty)}\left[C\left(A_{1}\right), C\left(A_{2}\right)^{-1}\right]$ is not Hurwitz.

Items (a) - (c) establish the following facts. Given two stable discrete-time second order LTI systems $\Sigma_{A_{1}}$ and $\Sigma_{A_{2}}$, a necessary condition for the existence of a CQLF is that the pencils $\sigma_{\gamma[0, \infty)}\left[C\left(A_{1}\right), C\left(A_{2}\right)\right]$ and $\sigma_{\gamma[0, \infty)}\left[C\left(A_{1}\right), C\left(A_{2}\right)^{-1}\right]$ are Hurwitz. Conversely, if a $\mathrm{CQLF}$ does not exist for $\Sigma_{A_{1}}, \Sigma_{A_{2}}$, then one of the pencils $\sigma_{\gamma[0, \infty)}\left[C\left(A_{1}\right), C\left(A_{2}\right)\right], \sigma_{\gamma[0, \infty)}\left[C\left(A_{1}\right), C\left(A_{2}\right)^{-1}\right]$ is not Hurwitz. Together these conditions yield the following result which is closely related to that presented in [11] and is the discrete-time counterpart of results presented in [10].

A necessary and sufficient condition for the stable discrete-time LTI systems $\Sigma_{A_{1}}$ and $\Sigma_{A_{2}}$, $A_{1}, A_{2} \in \mathbb{R}^{2 \times 2}$, to have a CQLF is that the pencils $\sigma_{\gamma[0, \infty)}\left[C\left(A_{1}\right), C\left(A_{2}\right)\right]$ and $\sigma_{\gamma[0, \infty)}\left[C\left(A_{1}\right), C\left(A_{2}\right)^{-1}\right]$ are Hurwitz.

\section{Concluding remarks}

In this paper we have derived a CQLF non-existence theorem. We have applied this theorem to derive a CQLF existence result for a pair of stable LTI systems that belong to a certain system class. We believe that our result can be applied to derive similar results for pairs of stable LTI systems belonging to other important system classes.

Acknowledgements: This work was partially supported by the European Union funded research training network Multi-Agent Control, HPRN-CT1999-00107 ${ }^{2}$ and by the Enterprise Ireland grant SC/2000/084/Y. Neither the European Union or Enterprise Ireland is responsible for any use of data appearing in this publication.

The authors gratefully acknowledge several discussions with Professor Kumpati S. Narendra.

\section{References}

[1] R. DeCarlo, M. Branicky, S. Pettersson, and B. Lennartson, "Perspectives and results on the stability and stabilizability of hybrid systems," IEEE Proceedings, vol. 88, no. 7, pp. 1069-1082, 2000.

\footnotetext{
${ }^{2}$ This work is the sole responsibility of the authors and does not reflect the European Union's opinion
} 
[2] H. Ye, A. N. Michel, and L. Hou, "Stability theory for hybrid dynamical systems," IEEE Transactions on Automatic Control, vol. 43, no. 4, pp. 461-474, 1998.

[3] R. N. Shorten and K. S. Narendra, "On the stability and existence of common Lyapunov functions for stable linear switching systems," in Proceedings of the 37th IEEE Conference on Decision and Control, 1998.

[4] R. N. Shorten and K. S. Narendra, "Investigating the stability of a class of hybrid system," IEE Computing and Control Engineering Journal, vol. 9, no. 2, pp.i81-88, 1998.

[5] M. Johansson and A. Rantzer, "Computation of piecewise quadratic Lyapunov functions for hybrid systems," IEEE Transactions on Automatic Control, vol. 43, no. 4, pp. 555-559, 1998.

[6] R. N. Shorten and F. O'Cairbre, "A new methodology for the stability. analysis of pairwise triangularisable and related switching systems," IMA Journal of Applied Mathematics. In Press.

[7] K. S. Narendra and J. Balakrishnan, "A common Lyapunov function for stable LTI systems with commuting A-matrices," IEEE Transactions on Automatic Control, vol. 39, no. 12, pp. 1669-1686, 1994.

[8] T. Ando, "Sets of matrices with a common lyapunov solution," Archiv der Mathematik, vol. 77, pp. 76-84, 2001.

[9] A. Agrachev and D. Liberzon, "Lie-algebraic stability criteria for switched systems," SIAM Journal of Control and Optimization, vol. 40, no. 1, pp. 253-269, 2001.

[10] R. N. Shorten and K. S. Narendra, "Necessary and sufficient conditions for the existence of a common quadratic lyapunov function in two stable second order linear systems," in Proceedings of American Control Conference, 1999.

[11] . M. Akar and K. S. Narendra, "On the existence of a common quadratic Lyapunov function for two stable second order LTI discrete-time systems," in Proceed. ings of American Control Conference, 2001.

[12] T. Ooba and Y. Funahashi, "On the simultaneous diagonal stability of linear discrete-time systems," Systems and Control Letters, vol. 36, pp. 175-180, 1999.

[13] Y. Mori, T. Mori, and Y. Kuroe, "A set of discrete-time linear systems which have a common quadratic Lyapunov function and its extension," in Proceedings of American Control Conference, 1998.

[14] W. J. Rugh, Linear System Theory. Prentice Hall, 1996.

[15] R. N. Shorten, K. S. Narendra, and O. Mason, "On common quadratic Lyapunov functions," IEEE Transactions on Automatic Control, vol. 48, no. 1, 2003.
[16] O. Mason and R. Shorten, "On common quadratic Lyapunov functions for stable discretetime LTI systems," tech. rep., NUI Maynooth, NUIM/SS/2002/05, 2002.

[17] R. Loewy, "On ranges of real Lyapunov transformations," Lin. Alg. and its Appl., vol. 13, pp. 79-89, 1976.

[18] G. Barker, A. Berman, and R. Plemmons, "Positive diagonal solutions to the Lyapunov equations," Linear and Multilinear Algebra, vol. 5, pp. 249-256, 1978.

[19] T. Kailath, Linear Systems. Prentice Hall, New Jersey, 1980.

[20] Z. Gajic and M. Qureshi, Lyapunov matrix equa tion in system stability and control. Academic Press, 1995. 